\title{
A perspective on paediatric surgical training: opportunities and challenges
}

\author{
N Patel, J Loveland \\ Department of Paediatric Surgery, University of the Witwatersrand \\ Corresponding author: Professor Jerome Loveland (loveland@wol.co.za)
}

S Afr J Surg 2018;56(1)

http://dx.doi.org/10.17159/2078-5151/2018/v56n1a2251

Globally, the training of paediatric surgeons is facing numerous challenges. Exposure to rare and complex index pathology is vital in the training of competent paediatric surgeons, but it is this rarity and complexity that creates a fundamental challenge to training programmes the world over. ${ }^{1}$ In the developing world, large paediatric populations facilitate trainee exposure to a wide variety of pathology and high case volume, but this training often occurs in settings in which treatment and teaching may not match the level of that in the developed world. ${ }^{1,2}$ In the developed world, training in paediatric surgery is under pressure from numerous fronts. Trainee work hour restrictions, the decreasing incidence of congenital abnormalities due to improved maternal screening programmes and funding directives resulting in simple paediatric surgical cases being treated outside of tertiary centres and thus out of reach of trainees have combined to decrease trainee exposure to rare and complex as well as and to common paediatric surgical pathologies. ${ }^{1}$

The majority of paediatric surgical conditions, for instance undescended testes and inguinal hernias, are relatively common and simple to manage. ${ }^{1}$ Nevertheless, the training of a complete paediatric surgeon also requires exposure to rare and complex surgical pathology, for instance oesophageal atresia, biliary atresia and anorectal malformations. ${ }^{1}$ Given that paediatric surgical training is still heavily dependent on the Halstedian apprenticeship model and the challenges facing training in paediatric surgery, many paediatric surgical trainees in the developed world are forced to move between hospitals, states and even countries to gain sufficient experience to be considered competent upon qualification. ${ }^{1}$ In developing countries, lack of well-structured training programmes in well-resourced facilities, relative shortage of ancillary services (for instance, specialised paediatric nurses, anaesthetists and intensivists), and overall shortage of health care workers and resources pose significant barriers to training. ${ }^{2}$ The paradox of the developing world with its relative oversupply of surgical pathology and severe shortage of human and other resources and the developed world with relative oversupply of human and ancillary resources but wherein exposure to complex and common paediatric surgical pathology is decreasing presents both challenges and opportunities for the training of skilled paediatric surgeons.

Competence in surgery encompasses proficiency in several aspects of patient management. While technical skill in the operating theatre is often used to distinguish one surgeon from another, competent paediatric surgeons are in fact required to be proficient in diagnosis, pre- and postoperative care, and working within a multi-disciplinary team. These skills may only be gained through time spent consulting and managing patients within an appropriate environment. It is well recognised within surgical and psychological literature that practice and experience are fundamental determinants of competence and excellence. This concept is known as 'deliberate practice' and holds that differences in performance between individuals can be explained by past and current levels of practice at a particular task or skill set. ${ }^{3,4}$ Malcolm Gladwell has built on this concept and defines 10000 hours as the minimum threshold of practice required in a particular activity in order to achieve technical excellence ${ }^{5}$ Notwithstanding the numerous divergent views within psychological and non-psychological literature around issues of deliberate practice, innate ability, self-motivation and other factors involved in becoming an expert in a particular field, it is widely acknowledged within the surgical fraternity that surgical competence requires an intensive investment of time by trainees within an appropriate environment in order to gain the skills, knowledge and maturity necessary to practice independently as professionals. It is within this 
context that the paediatric surgical community needs to attempt to address 3 key issues: (i) how to define high volume training, (ii) the relationship between high volume training and competence, and (iii) the association between competence and patient outcome.

Although optimal training and education in paediatric surgery is difficult to define, the traditional approach to surgical training has been Halstedian in nature. In fact, the founders of modern paediatric surgery, Ladd, Wyatt and Coe, are products of this model. ${ }^{6}$. This intensive approach defines a fixed period of training, educational content and patient exposure with increasing responsibility, and often requires residents to work in excess of 100 hours per week during their training. At its core, surgical training is experiential and exposure to a broad range of pathologies, perioperative care, critical care and nonoperative management of injury and illness is essential in the development of competence. ${ }^{7}$ Thus, whilst volume and years of training are only a proxy for competence, factors that mitigate exposure to patients inhibit resident education in surgical specialities. In the United States, surgical residents are expected to perform 750 surgical procedures in their 5 years of residency, including at least 150 procedures in their final year. ${ }^{8}$ Whilst minimum operative volumes in training are well defined, no such consensus exists for high volume training.

In 2003, the American Committee for Graduate Medical Education (ACGME) issued its first resident duty hour restrictions. These restrictions were instituted within residency programmes with the aim of improving patient safety, resident education and well-being, and professional identity. ${ }^{9}$ The ACGME restrictions were applied uniformly to all specialities, in spite of the opinions of surgical programme directors, who unlike their counterparts in paediatrics and medicine, were more likely to think that work hour restrictions would negatively impact on both resident training and patient care within their departments. ${ }^{9}$ To date, studies that have examined the effects of these work hour restrictions show mixed results on their effect on patient safety and resident well-being. As noted by Ziegler, ${ }^{6}$ although some studies show that the ACGME recommendations have improved the perceived quality of life of residents and the scores of junior residents in training examinations, they may have also resulted in decreased levels of patient satisfaction and increases in medical error, ostensibly due to a lack of continuity of care, and have even resulted in decreased resident operative volumes. In paediatric surgery, where programme accreditation in the United States is based on annual departmental and resident case volumes, work hour restrictions and subsequent decrease in trainee case volumes pose a significant threat to some training programmes. ${ }^{6}$ It should be noted that patient safety and resident education are dependent on more than resident working hours. An environment characterised by supervision customised to residents competence and one in which care is delivered in a team setting with multiple redundancies is vital to resident training and patient safety. ${ }^{10}$

In a survey of residents in general surgery, Spencer and Teitelbaum found that residents lost experience with outpatients in order to comply with working hour directives and maintain their operative case volumes. ${ }^{11}$ In a survey of paediatric surgeons in the United States, Ladd noted that one third of paediatric surgical trainees found that this also occurred in paediatric surgery. ${ }^{12}$ Further, $44 \%$ of paediatric surgical trainees found their opportunities to gain experience in affiliated subspeciality programmes was compromised in order to meet working hour directives. Paediatric surgical trainees also felt that working hour directives decreased opportunities for teaching of medical students and junior residents, as well as the didactic education of fellows. ${ }^{12}$ Only $50 \%$ of paediatric surgical fellows considered work hour restrictions to have improved their quality of life. ${ }^{12}$ This finding is supported by Nasca, who found that the time created by work hour restrictions is not necessarily being used by residents for study or sleep, and thus not enhancing resident education or reducing fatigue. ${ }^{10}$ The ACGME restrictions were revised in 2010 to include increased flexibility for senior trainees for exceptional clinical and educational circumstances, reductions in the length of the continuous duty period and stricter duty hour limits for junior trainees. Research on the impact of the revised restrictions on patient care and resident education is necessary and ongoing.

Notwithstanding the influence of other factors, such as natural aptitude, personality and environment, on the learning process, time spent engaging in a task is a critical determinant of an individual's aptitude in execution of that task. Nakayama notes that $25 \%$ of surgical graduates in the United States feel unprepared for independent practice upon completion of their residency, with $75 \%$ electing to undertake 1 to 2 year fellowships in order to gain the confidence, knowledge and skill required for independent practice. ${ }^{13}$ In another study, Nakayama notes that the average time spent in the operating theatre for surgical residents in the United States on completion of a 5-year residency was approximately 3500 hours, well short of the 10000 hours prescribed by Gladwell. ${ }^{13,5}$ In the context of paediatric surgical training, this practice needs to occur within the framework of a wellresourced and wellstructured training programme. While neither volume of operative cases, nor numbers of years in residency are proxies for surgical competence or excellence, time spent in intense training under appropriate supervision and with exposure to a wide range of pathologies and surgery is vital to (paediatric) surgical education. ${ }^{?}$

Within surgical literature, Birkmeyer has conclusively demonstrated that morbidity and mortality associated with certain procedures are indirectly related to both hospital and surgeon volume. ${ }^{14}$ In paediatric surgical literature, 
this relationship between surgeon volume and outcome and hospital volume and outcome has also been well documented by several authors with regards to simple (for instance, inguinal hernias) and complex (for instance, Kasai portoenterostomy) pathology. ${ }^{1,15,16}$ Clearly, whilst operative volume and time spent in training are not the only determinants of competence, they are imperative determinants of a trainee's ability to practice independently upon qualification. Given current challenges to the training of paediatric surgeons in the developed and developing world, the paediatric surgical community is required to reexamine training programmes in order to produce confident, competent, independent and indeed excellent paediatric surgeons. In the context of the current threats to the training of paediatric surgeons globally, potential solutions include: (i) accreditation of training centres based on annual case volume, (ii) rotation of trainees through high volume centres, and (iii) the introduction of novel training techniques, for instance modular training or web based techniques, or even restructuring of the service commitments of trainees.

Africa accounts for approximately $24 \%$ of the global disease burden. Distressingly, this load must be borne by $3 \%$ of the global health workforce on $1 \%$ of the global health budget. ${ }^{17}$ It is estimated that approximately $44 \%$ of the African population is between 0 and 14 years of age. ${ }^{17}$ Notwithstanding the approximately $250 \%$ increase in the number of paediatric surgeons (40 to 100) in Sub-Saharan Africa (SSA) between 2002 and 2008, the ratio of paediatric surgeons to children in Africa is still a remarkable 1 to 5 million. ${ }^{17}$ To aggravate this problem, most of the paediatric surgeons in SSA work in urban areas separating them from the rural population where the majority of children requiring access to paediatric surgeons live. ${ }^{18}$ Given the anticipated doubling of the African population by 2050 and subsequent 1 billion more children forecast to require access to surgical care, there is an urgent need to address the current and anticipated need for paediatric surgeons in Africa. ${ }^{17}$

Presently, the majority of surgical procedures carried out on children in Sub-Saharan Africa are performed by general surgeons and medical officers. ${ }^{17}$ This is in contrast to the developed world, and is contrary to a large body of existing evidence that clearly demonstrates decreased morbidity and mortality in children with simple and complex surgical pathologies managed by paediatric surgeons relative to those managed by general surgeons. ${ }^{1,15,16}$ In the African context, the problem may not be resolved by simply training more paediatric surgeons. Nevertheless, training of paediatric surgeons that set the standards for surgical care in children may serve as the catalyst for improvements in health care systems and the training of ancillary staff necessary to provide acceptable surgical care to children.

Looking specifically at the South African situation, the health care system of this emerging market affords numerous opportunities and challenges in the training of paediatric surgeons. Migration of qualified paediatric surgeons from developing to developed countries, the concentration of paediatric surgeons in urban areas, and the relative paucity of quality ancillary services are significant barriers to the widespread delivery of paediatric surgical care and training of paediatric surgeons beyond the major urban centres. That said, health care in these centres tends more towards that provided in high income countries. For example, the University of the Witwatersrand Paediatric Surgery Complex performs approximately 3500 operative procedures annually. The spectrum of pathology is extremely broad, including a relatively high incidence of complex index paediatric surgical cases. A subjective examination of the operative case volumes of foreign paediatric surgical trainees who have spent additional time training at our institution illustrates the glaring discrepancy between paediatric surgical training in the developed and the developing world. In one instance, in four years as a trainee in Europe an individual performed a single appendicectomy, 5 inguinal hernia repairs and was the assistant in 2 neonatal laparotomies. In 2 years as a trainee in South Africa, this individual was involved in 60 inguinal hernia repairs, 12 neonatal laparotomies, and 25 appendicectomies. Training in relatively well resourced high volume centres in the developing world provides a tremendous opportunity to train registrars in the surgical techniques and care required to produce competent paediatric surgeons capable of independent practice, and in addition provides adequate time and resources for embarking on the requisite clinical research.

In conclusion, training of paediatric surgeons is under threat the world over. Although the particular nature of these challenges varies from region to region, it is incumbent on the larger paediatric surgical community to respond. Defining high volume training, balancing trainee well-being with service provision, evaluating and utilising innovative training techniques, and utilising research and audits to tailor training programmes are essential steps that need to be taken in order to objectively restructure training with the goal of producing competent, and indeed excellent paediatric surgeons of the future.

\section{REFERENCES:}

1. Beasley SW. The challenges facing training in pediatric surgery worldwide. Front Pediatr. 2013;1:24. http://dx.doi. org/10.3389/fped.2013.00024

2. Butler MW, et al. The global paediatric surgery network: A model of subspecialty collaboration within global surgery. World J Surg. 2015;39. http://dx.doi.org/10.1007/s00268-0142843-1

3. Hambrick DZ, et al. Deliberate practice: Is that all it takes to become an expert? Intelligence. 2014;45. http://dx.doi. org/10.1016/j.intell.2013.04.001

4. Hambrick DZ, et al. Accounting for expert performance: The devil is in the details. Intelligence. 2014;45. http://dx.doi. org/10.1016/j.intell.2014.01.007 
5. Gladwell M. Outliers: The story of success. New York: Little, Brown and Co., 2008. Print.

6. Ziegler, M. Pediatric surgical training: An historic perspective, A formula for change. J Pediatr Surg. 2004;39. http://dx.doi. org/10.1016/j.jpedsurg.2004.04.002

7. Drake FT, et al. ACGME case logs: surgery resident experience in operative trauma for two decades. J Trauma Acute Care Surg. 2012;73(6). http://dx.doi.org/10.1097/TA.0b013e318270d983

8. Surgery Information: credit roles for surgery residents. 23 March 2016. Available from: http://www.acgme.org/ acgmeweb/tabid/377/ProgramandInstitutionalAccreditation/ SurgicalSpecialties/Surgery/CaseLoginformation.aspx

9. Antiel RM, et al. Duty hour recommendations and implications for meeting the ACGME core competencies: views of residency directors. Mayo Clin Proc. 2011;86(3). http://dx.doi. org/10.4065/mcp.2010.0635

10. Nasca TJ, et al. The new recommendations on duty hours from the ACGME task force. NEJM. 2010;363. http://dx.doi. org/10.1056/nejmsb1005800

11. Spencer AU, et al. The impact of work-hour restrictions on residents' operative volume on a subspeciality surgical service. J Am Coll Surg. 2005;200(5):670-6. http://dx.doi.org/10.1016/j. jamcollsurg.2005.01.008

12. Ladd AP. Pediatric surgery fellowship compliance to the 80-hour work week. J Pediatr Surg. 2006;41. http://dx.doi. org/10.1016/j.jpedsurg.2005.12.010

13. Nakayama DK. Getting to 10000 hours. J Surg Educ. 2013;71(2). http://dx.doi.org/10.1016/j.jsurg.2013.10.001

14. Birkmeyer JD, et al. Hospital volume and surgical mortality in the United States. NEJM. 2002;346(15). http://dx.doi. org/10.1056/NEJMsa012337

15. Borenstein $\mathrm{SH}$, et al. Effect of subspeciality training and volume on outcome after pediatric inguinal hernia repair. J Pediatr Surg. 2005;40(1). http://dx.doi.org/10.1016/j.jpedsurg.2004.09.002

16. Stringer MD. Biliary atresia: service delivery and outcomes. Semin Pediatr Surg. 2008;17(2). http://dx.doi.org/10.1053/j. sempedsurg.2008.02.007

17. Mars M. Paediatric surgery education in sub-Saharan Africa. Pediatric Surgery: A Comprehensive Text for Africa. Seattle: Global Help, 2010. p. 783-6. Print.

18. Krishnaswami $\mathrm{S}$, et al. The pediatric surgery workforce in low- and middle-income countires: problems and priorities. Semin Pediatric Surg. 2016;25. http://dx.doi.org/10.1053/j. sempedsurg.2015.09.007 